\title{
Artifact Removal Methods in EEG Recordings: A Review
}

\author{
Mariyadasu Mathe ${ }^{1,3, *}$, Padmaja Mididoddi ${ }^{2}$, Battula Tirumala Krishna ${ }^{1}$ \\ ${ }^{1}$ Department of Electronics and Communication Engineering, Jawaharlal Nehru Technological University Kakinada, \\ Andhra Pradesh, India \\ ${ }^{2}$ Department of Electronics and Communication Engineering, Velagapudi Ramakrishna Siddhartha Engineering College, \\ Andhra Pradesh, India \\ ${ }^{3}$ Gandhi Institute of Technology and Management, Andhra Pradesh, India \\ Received 10 May 2021; received in revised form 21 July 2021; accepted 22 July 2021
}

DOI: https://doi.org/10.46604/peti.2021.7653

\begin{abstract}
To obtain the correct analysis of electroencephalogram (EEG) signals, non-physiological and physiological artifacts should be removed from EEG signals. This study aims to give an overview on the existing methodology for removing physiological artifacts, e.g., ocular, cardiac, and muscle artifacts. The datasets, simulation platforms, and performance measures of artifact removal methods in previous related research are summarized. The advantages and disadvantages of each technique are discussed, including regression method, filtering method, blind source separation (BSS), wavelet transform (WT), empirical mode decomposition (EMD), singular spectrum analysis (SSA), and independent vector analysis (IVA). Also, the applications of hybrid approaches are presented, including discrete wavelet transform - adaptive filtering method (DWT-AFM), DWT-BSS, EMD-BSS, singular spectrum analysis - adaptive noise canceler (SSA-ANC), SSA-BSS, and EMD-IVA. Finally, a comparative analysis for these existing methods is provided based on their performance and merits. The result shows that hybrid methods can remove the artifacts more effectively than individual methods.
\end{abstract}

Keywords: EEG signal, canonical correlation analysis (CCA), SSA-BSS, EMD-IVA

\section{Introduction}

Electroencephalogram (EEG) is a voltage test recording the electrical activity of the neurons in the brain. EEG is obtained in various frequencies including delta $(1-4 \mathrm{~Hz})$, theta $(4-8 \mathrm{~Hz})$, alpha $(8-13 \mathrm{~Hz})$, beta $(13-30 \mathrm{~Hz})$, and gamma (greater than 30 $\mathrm{Hz}$ ) [1]. EEG brain rhythms with different frequency ranges are shown in Table 1 [2]. EEG is suitable for brain signal acquisition because of its non-intrusiveness, portability, high temporal resolution, and cost-effectiveness. Brain signal acquisition and processing are mainly used for clinical research purposes to diagnose multiple brain disorders, e.g., sleep disorders, depression, schizophrenia, and epileptic disorder [3]. However, EEG processing and analysis are affected by a couple of conditions, such as technical and physiological conditions. During the signal acquisition process, the most prevalent disorders (artifacts) are ocular, muscle, and cardiac disorders, and electrooculogram (EOG) is cumbersome [4].

Table 1 Basic brain rhythms and their frequency range [2]

\begin{tabular}{|c|c|c|c|}
\hline Serial number & Brain rhythm & Frequency range & State of the brain \\
\hline 1 & Delta & $1-4 \mathrm{~Hz}$ & Sleeping/unconscious \\
\hline 2 & Theta & $4-8 \mathrm{~Hz}$ & Imagination \\
\hline 3 & Alpha & $8-13 \mathrm{~Hz}$ & Calm consciousness \\
\hline 4 & Beta & $13-30 \mathrm{~Hz}$ & Focused consciousness \\
\hline 5 & Gamma & $>30 \mathrm{~Hz}$ & Peak performance \\
\hline
\end{tabular}

* Corresponding author. E-mail address: mmathe@ gitam.edu 
These artifacts, power line interference, impedance fluctuation, and wire movement collide and intrude the energy with EEG signals. Due to these artifacts, it is difficult to obtain the correct diagnosis and analysis of EEG. Therefore, artifact removal methods are required to reduce the artifacts and preserve the originality of the actual EEG signals. The placement of electrodes in the 10-20 system is shown in Fig. 1.

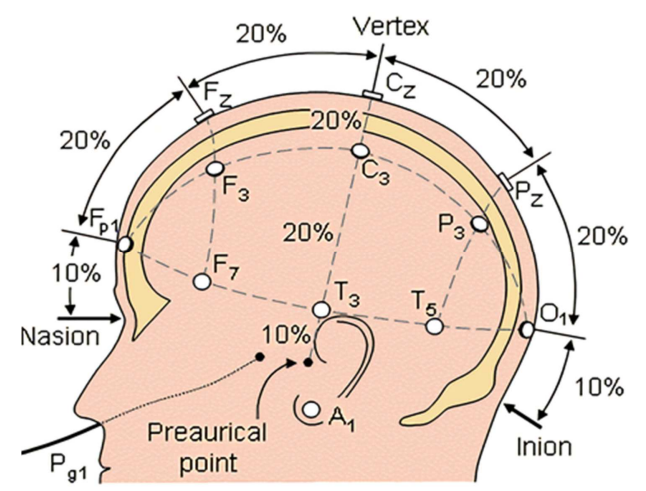

Fig. 1 Depicture of 10-20 electrode placement system [5]

Researchers developed automated approaches and techniques wherein the EEG signals are recorded using single- and multi-channels. Different methods are proposed for the mitigation of artifacts from single- and multi-channels. The accuracy of the methods using single-channels is less than the one using multi-channels, but the complexity over time is less when using single-channels. The basic process includes recording the EEG data with precaution and removing the epochs in EEG data, which can cause the removal of some valuable data [6]. Generally, artifact removal techniques are categorized into two types: regression-based method and blind source separation (BSS) based method [7]. So far, no specific method can remove all the artifacts. Researchers are trying to suppress the artifacts by improving the existing techniques and combing two and more methods to get the artifact-minimized EEG signals.

The main objective of this study is to give an overview on the existing methodology to remove the artifacts in EEG recordings and to choose the better method based on artifact removal types. The remaining paper is organized as follows. The artifacts affecting the EEG recordings are discussed in section 2. Literature review is clearly explained in section 3. The primary artifact removal techniques' advantages and disadvantages proposed by various researchers are discussed in section 4 . The comparative analysis for multiple artifact removal methods is presented in section 5 .

\section{About the Artifacts}

Human brain is a combination of billions of neurons, and the activity of this brain region is measured by recording the electrical signals generated by the neurons. EEG is such a signal recording acquired by placing the electrodes on the scalp. The recorded EEG signals include the signals from the brain neurons and the neurons of other body parts. The signals generated by other than brain neurons are called artifact signals. These artifacts are due to ocular artifacts (OAs), muscle artifacts, cardiac artifacts, electrode placement, impedance mismatching, etc. [8]. Non-physiological artifacts can be removed by simple methods such as high-pass filtering and low-pass filtering because these artifacts' frequency does not overlap with the desired EEG signals [9]. However, the removal of physiological artifacts requires different methods because the frequency spectrum of physiological artifacts overlaps with the desired EEG signals [10].

OAs are generated from eye movements (EMs) and eye blinks, and are propagated to the scalp region and overlap with the EEG signals. The OA frequency range is petite and is recorded by EOG by placing the electrodes (one above the eye and the other on one side of the eye) as shown in Fig. 2. EEG signals are deteriorated by EOG signal components and vice versa, so bi-directional interference is introduced [11]. The amplitude of EOG is higher than the amplitude of EEG. Muscle artifacts are originated from different muscle groups such as contraction, stretch, talking, sniffing, swallowing, etc. The frequency range of 
muscle artifacts is vast and is overlapped entirely with the actual EEG signals. The amplitude of muscle artifacts depends on the activity of the muscle and is measured by electromyography (EMG). Because of the broad spectrum, muscle artifacts cannot be obtained by single-channels. The autocorrelation of muscle artifacts is very small and independent of the EEG signals both temporally and spatially [8]. Cardiac artifacts are originated from the heart and are measured by electrocardiography (ECG). Cardiac artifacts follow some regular patterns, so removing the cardiac artifacts is simple by using reference signals.

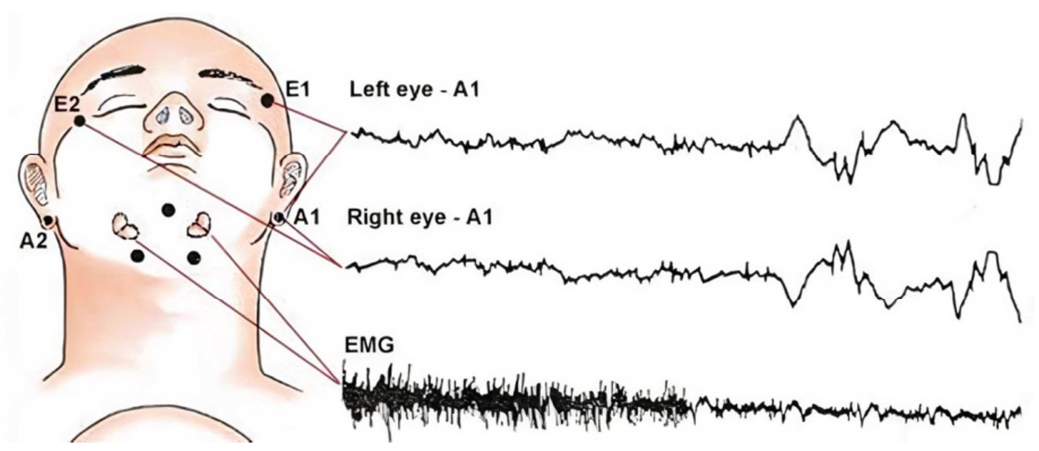

Fig. 2 EOG and EMG elements of a sleep montage setup [5]

For the better analysis of human brain and the diagnosis of patients, artifacts must be removed. Generally, the recorded EEG signals are between the ranges of $0.01 \mathrm{~Hz}$ to $100 \mathrm{~Hz}$. Different types of the brain rhythms and the artifacts generated by the brain and body are shown in Fig. 3 and Fig. 4.

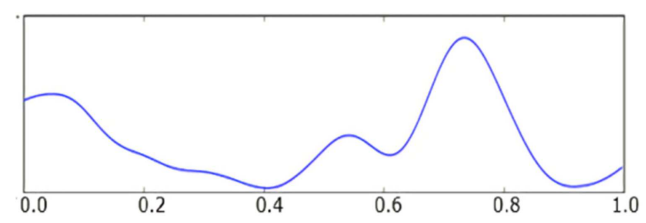

(a) Delta wave

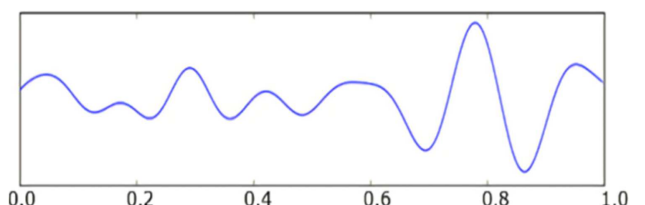

(b) Theta wave

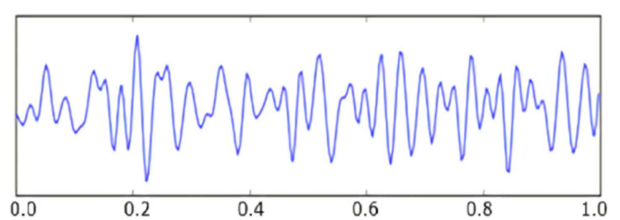

(d) Beta wave

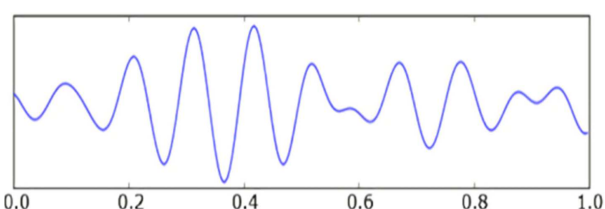

(c) Alpha wave

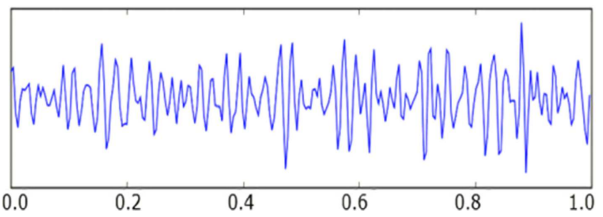

(e) Gamma wave

Fig. 3 Graphical representation of different brain rhythms

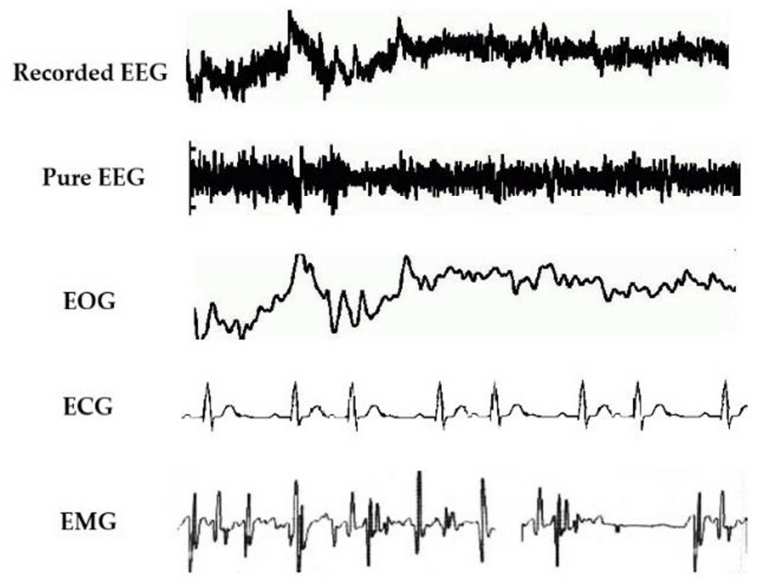

Fig. 4 Different physiological artifacts [8] 


\section{Literature Survey}

\subsection{Related works}

In 2013, Zou et al. [12] have addressed an automatic algorithm for general artifact identification. It was composed of electrode-scalp impedance information and an event-associated feature-oriented clustering algorithm. The former identified the non-biological artifacts, and the latter identified the artifacts with physiological origins. It could efficiently remove, separate, and detect the non-biological and physiological artifacts. It efficiently improved the signal quality. The classification accuracy was also improved. Mahajan and Morshed [13] have proposed a uniquely robust, unsupervised, and computationally speed statistical algorithm that employed modified multiscale sample entropy (mMSE) and kurtosis to recognize the independent eye blink artifactual components automatically. These components were denoised by the biorthogonal wavelet decomposition. The persistent neural activity was preserved in the independent components (ICs), and the artifactual activity was removed. Their proposed method attained average specificity, sensitivity, and execution time, which are better than the ones in other previously existing methods.

In 2014, Acharjee et al. [14] have described the conflict of gradient artifact removal from the EEG signals recorded in a concurrent manner with functional magnetic resonance imaging (MRI) acquisition. The artifacts were estimated by the quasi periodicity and its similarity over distinct channels with the help of independent vector analysis (IVA). Their proposed method utilized spatio-temporal information to evaluate the artifacts for a specific channel with the support of spatial dependences. Hence, it offered robustness in terms of uncontrollable variations such as fluctuations and head movement during acquisition. In 2015, Khatun et al. [15] have evaluated the unsupervised wavelet transform (WT) approach for removing OAs in the case of a single-channel EEG system. A group of 7 raw EEG dataset was investigated. Two familiar WT techniques, i.e., stationary wavelet transform (SWT) and discrete wavelet transform (DWT), were used. Four WT basis functions were utilized for removing OAs, i.e., bior4.4, sym3, coif3, and haar, having statistical threshold (ST) and universal threshold (UT). Their proposed method was an efficient tool for real-time applications.

In 2016, Tibdewal et al. [16] have performed detection of EM artifacts and classification of non-artifactual/artifactual EEG time series together with multiple EM artifactual zones. Artificial neural network (ANN) was employed for the identification. The researchers also proposed a computationally fast, robust, simple, and novel time-amplitude algorithm. Their proposed method provided the ANN model accuracy for the classification of the artifacts, and detected and marked the multiple EM artifactual zones automatically and appropriately. Liu et al. [17] have introduced a practical and efficient filtering algorithm based on multiscale entropy and multivariate empirical mode decomposition (MEMD) for computing the depth of anesthesia (DOA). The mean entropy found the artifact-free intrinsic mode functions (IMFs). The impact of distinct levels was investigated by means of the simulated data. The complexity was also computed for monitoring the DOA. The ANN was used for the range mapping for correlating the measurements with bispectral index (BIS). The BIS and entropy values contained a more robust correlation, and it also efficiently filtered the artifacts.

In 2017, Barua et al. [18] have proposed an algorithm which is known as automated artifact handling in EEG (ARTE). It was employed as a pre-processing step in the driver monitoring application, and functioned based on the wavelets, hierarchical clustering, and independent component analysis (ICA). It was analyzed on a driver sleepiness study, and was evaluated with the help of a clinical neurophysiologist using quantitative measures. The outcomes demonstrated that it minimized the effect of artifacts in the recorded EEG signals. It was a data-driven one, and it did not depend on manually defined thresholds or extra reference signals. Sai et al. [19] have introduced a new technique for artifactual component identification with the help of a pre-trained support vector machine (SVM). The researchers also proposed an extendable and robust system that enabled removing the artifacts without arbitrary thresholding. The target artifacts were removed in a successful manner. The training 
used a group of features such as amplitude range, Shannon's entropy, variance, and kurtosis. The multiple forms of artifacts in the multi-channel EEG were also accommodated. Li et al. [20] have introduced a unified framework based on canonical correlation analysis (CCA) to remove artifacts.

The raw signals constructed a pair of matrices based on the sources via the autocorrelation maximization. These sources were eradicated by placing them as zeros, and the remaining sources reconstructed the artifact-free EEG. The actual and simulated recorded data assessed this framework. It lessened the associated motion artifacts and contained benefits for eliminating the gradient artifacts with respect to the computational complexity and combination of characteristics. Roy and Shukla [21] have compared the effectiveness of enhanced empirical mode decomposition (EEMD) with distinct interpolations based on an artifact removal technique. The input single-channel EEG signals were transformed to multi-channel signals, and the motion artifact randomness was eradicated by DWT filtering and CCA. Their proposed method acted as a supplement for the traditional algorithms and provided enhancements in difference signal to noise ratio (DSNR) and several performance parameters.

In 2019, Chang et al. [22] have evaluated artifact subspace reconstruction (ASR) during the simulated driving experiments. The IC classifier and ICA separated the artifacts for assessing the efficiency of ASR. The temporal activity power was minimized. It enhanced the quality of a subsequent ICA decomposition. The brain activities were retained, and non-brain signals were removed. Their proposed method was an automatic and powerful artifact removal technique for online real-time EEG applications or offline data analysis like brain-computer interfaces and clinical monitoring. Cheng et al. [23] have addressed an efficient technique for removing different artifacts for the single-channel EEG. It was a combination of second-order blind identification and singular spectrum analysis (SSA) method. The possible cases contaminated by ECG, EOG, and EMG artifacts were analyzed. Their proposed method removed different artifacts and acted as a promising tool for biomedical signal processing applications. Saini et al. [24] have introduced a robust framework for detecting and eliminating OAs based on turning point count and variational mode decomposition (VMD). It exploited the efficiency in two stages, i.e., VMD-I and VMD-II. It was composed of four components: EEG signal decomposition into two modes using VMD-I, rejection of low-frequency baseline components, processed EEG signal decomposition into three modes using VMD-II, rejection of mode containing OAs based on turning point count-based threshold criteria.

The OAs were eliminated with minimal loss in the entire local rhythms and reconstructed EEG signals. Liu et al. [25] have labeled a robust and effective muscle artifact removal technique with the help of fast multivariate empirical mode decomposition (FMEMD) and CCA in the case of few-channel EEG. The input EEG recordings were decomposed into various multivariate IMFs. The CCA processed the IMFs for measuring the underlying sources. In the final step, the sources with less autocorrelations were eradicated. The average correlation coefficient (CC) and the accuracy were largely consistent. Their proposed method worked much robust in the low sampling rate based on the benchmark and real data. Dora and Biswal [26] have suggested an ECG artifact correction algorithm for the automatic diagnosis and analysis of single-channel EEG signals. It employed a modified and enhanced signal decomposition version for attaining band limited intrinsic mode functions (BLIMFs). It was helpful when the signal was composed of correlated properties. It suppressed the QRS complexes and estimated the ECG reference. It offered less distortion and was computationally more intensive than other methods. It overcomes the shortcomings such as the requirement of a reference ECG channel and the requirement of R-R interval or amplitude thresholding for QRS complex identification.

In 2020, Dora et al. [27] have introduced an adaptive SSA algorithm for muscle artifact removal. The mobility threshold was decided in an adaptive manner by neural network regressor (NNR). Their proposed method was trained using several contamination levels. It discriminated among several contamination levels and performed superior to the traditional single-channel algorithms. Yedurkar and Metkar [28] have proposed a technique for removing physiological artifacts and 
positioning the epileptic region. A hybrid method was recommended based on multi-resolutional analysis and adaptive filtering (MRAF) . In the initial step, the EEG signals were decomposed by DWT that localized the epileptic region. The multi-resolutional soft thresholding removed the abrupt changes.

Moreover, the adaptive filtering (AF) eradicated the low-frequency components that described the physiological artifacts. It retained most of the seizure signals that were available in the validated datasets. Islam et al. [29] have introduced a technique for removing motion-related artifacts in the field of epilepsy. Initially, it involved the EEG signal recording with the help of a wearable EEG headset. It was colored using few motion artifacts produced in a lab-controlled experiment. It was followed by the spectral and temporal spectral signal characterization and artifact removal by ICA. The actual clinical EEG data tested it, and the outcomes described an average improvement in accuracy for detecting the seizures and prediction. Bajaj et al. [30] have addressed an algorithm based on wavelet packet decomposition (WPD) that permitted the presumed artifact removal by optimizing intuitive parameters. It was composed of 2 tuning parameters and 3 operating modes. It was compared with comparative wavelet-oriented technique and ICA-oriented techniques. The performance could be enhanced by proper optimization of the parameters for an individual predictive method. It was accomplished better for artifactual noise removal and neural information retention computed using CC and mutual information (MI) than the comparative wavelet-oriented technique.

In 2021, Noorbasha and Sudha [31] have labeled the conflict of EOG artifact removal and separation of different cerebral activities in a single-channel contaminated EEG. A new method based on ICA and SSA together with SWT was proposed. The single-channel contaminated EEG signals were transformed into multivariate information. Then, ICA separated the source signals as distinct ICs. The SWT accomplished the thresholding for preserving the EEG signals and dividing the actual artifacts. Their proposed method provided better artifact separation characteristics than the traditional approaches.

\subsection{Utilized datasets}

Several kinds of datasets are used in the considered previous research, including the manual dataset, Mendeley, MIT-BIH polysomnographic database, EEG during mental arithmetic task database, synthetic dataset, simulated dataset, temple university hospital (TUH) EEG database, gold-standard dataset, epileptic EEG signal dataset from the local hospital, Freiburg EEG dataset, semi-simulated dataset, real dataset, open-source dataset, etc. Table 2 lists different kinds of datasets used in the considered works in a detailed manner. Most of the considered works utilize the manual dataset, followed by a real dataset, simulated dataset, MIT-BIH polysomnographic database, etc. The manual dataset is used in 50\% of the works, real dataset was used in $20 \%$ of the works, simulated dataset was used in $15 \%$ of the works, MIT-BIH Polysomnographic database was used in $10 \%$ of the works, and the remaining datasets was used only in $5 \%$ of the works.

Table 2 Different datasets used in the considered works

\begin{tabular}{|c|c|}
\hline Dataset & Previous work \\
\hline Manual dataset & {$[12-15,18-19,22-23,25,31]$} \\
\hline Mendeley & {$[24]$} \\
\hline MIT-BIH polysmnographic database & {$[24,26]$} \\
\hline EEG during mental arithmetic task database & {$[24]$} \\
\hline Synthetic dataset & {$[31]$} \\
\hline Simulated dataset & {$[27]$} \\
\hline TUH EEG database & {$[28]$} \\
\hline Gold standard dataset & {$[28]$} \\
\hline Epileptic EEG signal dataset from local hospital & {$[29]$} \\
\hline Freiburg EEG dataset & {$[26]$} \\
\hline Semi-simulated dataset & {$[16-17,20,30]$} \\
\hline Real dataset & {$[21]$} \\
\hline Open-source dataset & \\
\hline
\end{tabular}




\subsection{Simulation platforms}

The platforms used in the considered works are given in Fig. 5. Here, MATLAB and LabVIEW softwares are used for the implementation. $92 \%$ of the works use the MATLAB software, and $8 \%$ use the LabVIEW software.

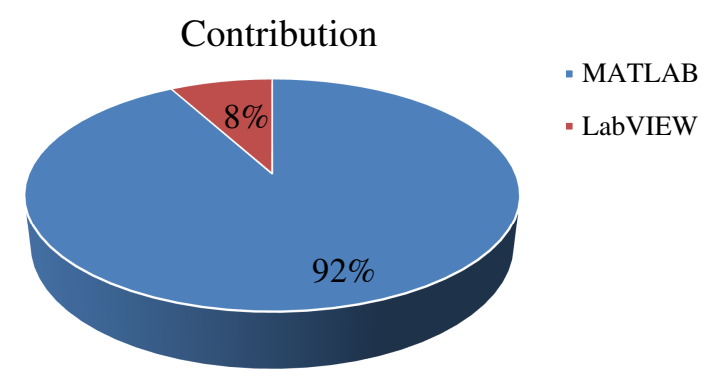

Fig. 5 Simulation platforms used in the literature

\subsection{Performance measures}

The performance measures used in the analyzed research are depicted in Table 3, including accuracy, sensitivity, specificity, mean square error (MSE), root mean square error (RMSE), relative root mean square error (RRMSE), etc. Among the considered works, time measure is used in $25 \%$ of the works, CC is used in $45 \%$ of the works, MI is used in $25 \%$ of the works, accuracy is used in $20 \%$ of the works, sensitivity is used in $25 \%$ of the works, specificity is used in $15 \%$ of the works, MSE is used in $10 \%$ of the works, mean absolute error (MAE) is used in $20 \%$ of the works, RRMSE is used in $25 \%$ of the works, RMSE is used in $15 \%$ of the works, signal to noise ratio (SNR) is used in $30 \%$ of the works, power spectral density (PSD) is used in $10 \%$ of the works, signal to artifact ratio (SAR) is used in $10 \%$ of the works, and the remaining measures such as euclidean distance, normalized mean square error (NMSE), spectral coherence, agreement rate, signal quality index (SQI), relative error (RE), normalized root mean square error (NRMSE), variance, power, maximum absolute error (MAX), normalized maximum absolute error (NMAX), normalized root mean square difference (NRD), percentage root mean square difference (PRD), autocorrelation, peak signal to noise ratio (PSNR), precision, false alarm rate (FAR), coherence, mean entropy value (MEV), DSNR, and spectral distortion improvement are used only in 5\% of the contributions.

Table 3 Performance measures used in the previous research

\begin{tabular}{|c|c|c|c|c|c|c|c|c|c|c|c|c|c|c|}
\hline Ref. & Time & $\mathrm{CC}$ & MI & Accuracy & Sensitivity & Specificity & MSE & MAE & RRMSE & RMSE & SNR & PSD & SAR & Miscellaneous \\
\hline [12] & $\sqrt{ }$ & - & - & - & - & - & - & - & - & - & - & - & - & Euclidean distance \\
\hline [13] & $\sqrt{ }$ & $\sqrt{ }$ & $\sqrt{ }$ & $\sqrt{ }$ & $\sqrt{ }$ & $\sqrt{ }$ & - & - & - & - & - & - & - & $\begin{array}{l}\text { Spectral coherence } \\
\text { and agreement rate }\end{array}$ \\
\hline [14] & - & - & $\sqrt{ }$ & - & - & - & - & - & - & - & - & - & - & - \\
\hline [15] & - & $\sqrt{ }$ & $\sqrt{ }$ & - & - & - & - & - & - & - & - & - & $\sqrt{ }$ & NMSE \\
\hline$[16]$ & - & - & - & - & - & - & - & $\sqrt{ }$ & - & - & - & - & - & - \\
\hline [17] & $\sqrt{ }$ & - & - & - & - & - & - & - & - & - & $\sqrt{ }$ & - & - & MEV \\
\hline$[18]$ & - & - & - & - & - & - & $\sqrt{ }$ & $\sqrt{ }$ & $\sqrt{ }$ & - & - & - & - & SQI, RE, and NRMSE \\
\hline [19] & - & - & - & $\sqrt{ }$ & $\sqrt{ }$ & $\sqrt{ }$ & - & - & - & - & - & - & - & - \\
\hline [20] & - & $\sqrt{ }$ & - & - & - & - & $\sqrt{ }$ & - & - & - & $\sqrt{ }$ & - & - & - \\
\hline [21] & - & - & - & - & - & - & - & - & - & $\sqrt{ }$ & - & - & - & $\begin{array}{c}\text { DSNR and spectral } \\
\text { distortion } \\
\text { improvement }\end{array}$ \\
\hline$[22]$ & - & - & - & - & - & - & - & - & - & - & - & - & - & Variance and power \\
\hline [23] & - & $\sqrt{ }$ & - & - & - & - & - & - & $\sqrt{ }$ & $\sqrt{ }$ & $\sqrt{ }$ & - & - & - \\
\hline [24] & $\sqrt{ }$ & $\sqrt{ }$ & - & - & - & $\sqrt{ }$ & - & $\sqrt{ }$ & - & $\sqrt{ }$ & $\sqrt{ }$ & - & - & $\begin{array}{c}\text { MAX, NMAX, NRD, } \\
\text { and PRD }\end{array}$ \\
\hline [25] & $\sqrt{ }$ & $\sqrt{ }$ & - & - & - & - & - & - & $\sqrt{ }$ & - & - & $\sqrt{ }$ & - & Autocorrelation \\
\hline [26] & - & $\sqrt{ }$ & - & - & - & - & - & - & - & - & $\sqrt{ }$ & $\sqrt{ }$ & $\sqrt{ }$ & - \\
\hline [27] & - & $\sqrt{ }$ & $\sqrt{ }$ & - & - & - & - & - & $\sqrt{ }$ & - & - & - & - & PSNR \\
\hline [28] & - & - & - & $\sqrt{ }$ & $\sqrt{ }$ & $\sqrt{ }$ & - & - & - & - & - & - & - & Precision \\
\hline [29] & - & - & - & $\sqrt{ }$ & $\sqrt{ }$ & - & - & - & - & - & - & - & - & FAR \\
\hline [30] & - & $\sqrt{ }$ & $\sqrt{ }$ & - & - & - & - & - & - & - & - & - & - & Coherence \\
\hline [31] & - & - & - & - & - & - & - & $\sqrt{ }$ & $\sqrt{ }$ & - & $\sqrt{ }$ & - & - & - \\
\hline
\end{tabular}




\section{Survey on the Existing Artifact Removal Techniques}

\subsection{Artifact avoidance and rejection}

The first basic technique to remove artifacts is to instruct the patient to shun unnecessary actions such as EMs and stay calm until the completion of the process. However, since it is not possible to keep quiet, artifacts are recorded in the EEG signals [3]. The second way is to remove the epochs in EEG data by observation. This method is also not available because the data is vast, and the expertise must be analyzed appropriately. Therefore, a separate automatic procedure is needed to remove the artifacts as much as possible [32].

\subsection{Regression methods}

The regression method is a straightforward and commonly used method for removing artifacts in EEG signals. In this method, a reference signal is required to remove artifacts. EEG signals are generally most affected by EOG and EMG signals. The regression method depends on the subtraction of artifact signals from the EEG signals. It assumes that OAs and EEG signals are linearly uncorrelated [33]. Identifying the reference artifact signals is very difficult because EEG and EOG are both neural activities [2]. While subtracting EOG signals, some useful information related to the original EEG signals was also removed; loss of data is there. The simple mathematical analysis is shown here in Eq. (1).

$$
E E G_{s}(l)=E E G_{o}(l)+\gamma E O G(l) ; \text { with } l=1,2, \cdots, O
$$

Here, the recorded EEG signals are denoted as $E E G_{s}$ the fraction of the EOG signals is denoted as $\gamma$, and the original EEG signals are denoted as $E E G_{o}$ respectively.

\subsection{Filtering methods}

\subsubsection{Adaptive filtering}

The performance of WT depends upon the mother wavelet effectively. The WT efficiency is improved if the artifact component's frequency does not overlap with the usage frequency of the EEG signals. Unfortunately, ECG, EMG, and EOG artifact frequencies are coinciding with the desired EEG signals. In ICA, the improvement performance of artifact removal depends on the reference signal and a large amount of data. However, AF overcomes the problem of extensive data and frequency band overlap [34]. Fig. 6 presents a block diagram to implement AF.

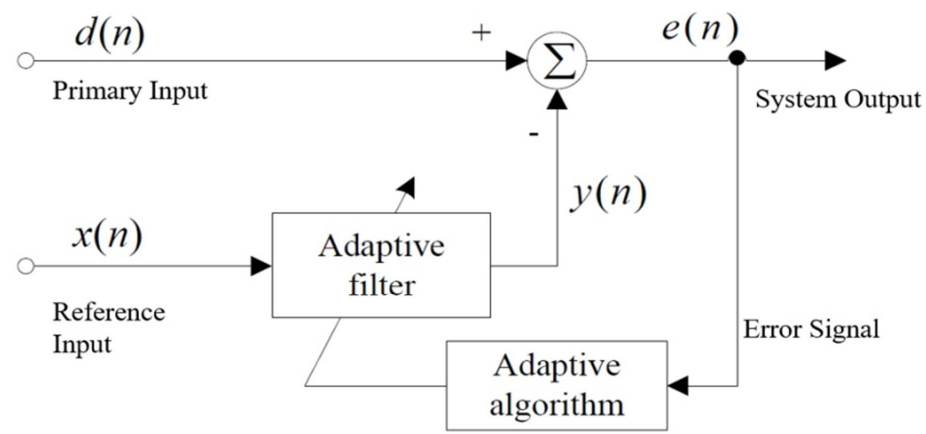

Fig. 6 Basic block diagram of adaptive filter [34]

More than one channel can be selected as a reference channel, and AF is employed mainly to minimize power line interference artifacts. However, this has two particular limitations: (i) the noise content in the original signals must be corrected with some reference noise signals; (ii) the original signal helpful information may be uncorrected with the reference noise. 


\subsubsection{Kalman filtering}

Kalman filter (KF) is one of the appropriate estimators for the analysis of linear systems and signals. It is a recursive filtering algorithm to remove the artifacts in EEG signals. The inputs used for this algorithm are the state of the system, recorded signal, and past observations. In this algorithm, a reference signal is not required; KF estimates the state of the system and the reference signal from the contaminated part of the EEG signals. The recorded EEG signals are denoted as $E E G_{r}$, true EEG signals are denoted as $E E G_{t}$, and OAs are denoted as $E O G$. KF uses a state-space model to represent the complete analysis. The state-space model of the KF is as follows [35]:

$$
\begin{aligned}
& x(n)=A x(n-1)+B u(n-1)+C w(n-1) \\
& z(n)=H x(n)+v(n)
\end{aligned}
$$

where $z(n)$ is the measured signal; $x(n)$ is the system's state; $A, B, C$, and $H$ are the matrices obtained from different regions of the acquired EEG signals; $w(n)$ and $v(n)$ are the process noise and measurement noise respectively; $u(n)$ is the control input for the system. The measured signal is the combination of $E E G_{t}$ and artifactual signals like EOG [36].

$$
z(n)=E E G_{t}+E O G=H x(n)+v(n)
$$

After estimating EEG and EOG components, KF is applied to remove the artifacts. In this algorithm, two steps are there.

(1) Estimate the state variables.

(2) Compare the previous estimation with the measured signal to obtain an improved version of the state variables.

The disadvantage of KF is that it assumes the errors are Gaussian, and the system is a linear model. The measurement of covariance matrices for process noise and measured noise is tough to calculate because the practical recorded EEG signals are not a linear combination of the underlying source signals. The extension of KF is extension Kalman filter (EKF) and unscented Kalman filter (UKF), which work on nonlinear systems.

\subsection{Blind source separation (BSS)}

BSS is a separation approach of underlying sources from the mixed signals without knowing prior information about the origin of signals and mixing parameters. The main plan of BSS is to recover the sources from mixed signals [37]. Let us consider $X$ as an observed EEG signal from the scalp electrodes, which is a linear mixture of underlying sources $(S)$ with unknown mixing matrix $A$, Eq. (5) is obtained.

$$
X=A S
$$

BSS algorithm is reverted to recover the underlying sources.

$$
Y=W S
$$

where $Y$ is the estimation of sources, and $W$ is an un-mixing matrix. These algorithms are used to remove artifact components and to reconstruct artifact-free EEG signals. In the following section, some of the BSS algorithms are discussed.

\subsubsection{Principle component analysis $(P C A)$}

Principle component analysis (PCA) is one of the simple data reductions and BSS techniques to remove the artifacts using the orthogonality principle [8]. Berg and Scherg proposed PCA to minimize OAs. PCA is a multiple data analysis procedure that involves the transformation of many correlated variables into a smaller number of uncorrelated variables. PCA is defined 
as "linear projection that transforms multivariate data into a set of components called principle components (PCs)" [38]. PCs are calculated using a simple algorithm called singular value decomposition (SVD). In SVD, eigenvalues are computed from the covariance matrix $(C)$ [36]. Most significant eigenvalues are related to the highest variance, and the lowest eigenvalues are related to the lower variance. Assume the recorded EEG signals $\left(E E G_{r}\right)$ combine the original EEG signals $\left(E E G_{o}\right)$ and the artifacts from EOG and EMG, Eq. (7) is obtained.

$$
E E G_{r}=E E G_{o}+\alpha \times E O G+\beta \times E M G
$$

where $\alpha$ and $\beta$ are real constants. The drawback of PCA is the orthogonality principle only because it cannot separate the artifacts if they have the same amplitude as original EEG data. The orthogonality principle is not always held due to the nonlinearity between the electrical signals generated by brain neurons from eye blinks and EMs compared with original EEG signals [39]. The advancement in PCA is done by robust PCA and kernel PCA [40]. The performance of PCA is better compared to the regression method and filtering methods. PCA can remove OAs, but other artifacts cannot be removed. Therefore, researchers preferred alternative methods such as ICA, CCA, empirical mode decomposition (EMD), IVA, SSA, etc.

\subsubsection{Independent component analysis (ICA)}

ICA is a powerful BSS method to remove the artifacts in EEG signals. It converts the multi-dimensional data into different ICs. In this method, the observed EEG signals' primary assumption is a linear mixture of underlying independent sources [39]. PCA is unable to differentiate the artifacts if the neural activity and artifacts have the same amplitude. Assume that the recorded EEG signals $X(k)$ use $N$ channels and that the number of underlying sources $S(k)$ are $N$ [2], Eq. (8) is obtained.

$$
X(k)=A S(k)
$$

where $X(k)=\left[X_{1}(k), X_{2}(k), \ldots, X_{\mathrm{N}}(k)\right]^{T}$ and $S(k)=\left[S_{1}(k), S_{2}(k), \ldots, S_{\mathrm{N}}(k)\right]^{T} . T$ and $k$ are transpose operator and time index operator, respectively. $A \epsilon R^{N X N}$ is mixing the unknown matrix. The goal of ICA is to recover $S$ from $X$ without knowing $A$ and $S$. For this, a separate un-mixing matrix $W$ should be found such that Eq. (9) is obtained.

$$
Y(k)=W X(k)
$$

where $Y$ is the expected value of $S . Y$ is precisely equal to $S$ if and only if $W=A^{-1}$. This model is by assuming no noise model. For experimental conditions, some noises are also included. A simple model of how the electrodes are placed to record the EEG signals from the brain is shown in Fig. 7.

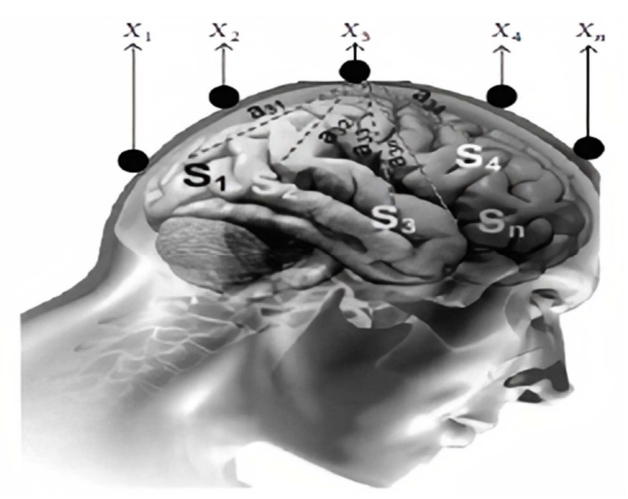

Fig. 7 A simple model of recording EEG signals by electrodes [2]

In the preprocessing of EEG signals using ICA, two main steps are there; one is centering, and the second is whitening [37]. In the centering step, the EEG data is centered by subtracting the mean from the data. In the whiting step, the EEG data is de-correlated. ICA uses higher-order statistics (HOS) to identify independent artifactual components. Unlike PCA, 
orthogonality is not required; only statistical independence is needed. The main drawback of ICA is that the number of channels used to collect the data should be huge compared to underlying artifactual sources. The second drawback is that ICA removes the artifacts whose topologies are stereotyped like EOG, but muscle artifacts are not stereotyped because the muscle artifacts are from different sources.

\subsubsection{Canonical correlation analysis (CCA)}

Hotelling in 1936 has proposed CCA. The entire concept of this approach is to find the vectors for two dataset variables so that the correlation between the projections of the variables on this basis vector is mutually maximized. This analysis relies on the coordinate system where the variables are described; however, if there is a strong linear relationship between the sets of multi-dimensional variables, they may depend on the coordinate system. This correlation relation may not be visible, but it may seek a pair of linear transformations. Each set of variables are transformed, and the corresponding coordinates are correlated to the maximum [41]. CCA is also treated as one of the powerful tools used in BSS to remove artifacts in EEG signals. This analysis mainly uses second-order statistics (SOS) with a less computational cost than ICA (which uses HOS) [42]. CCA removes muscle artifacts easily because muscle artifacts do not have stereotyped topography [43]. Muscle artifacts are distributing a total range of frequency spectrum. The autocorrelation of muscle artifacts is very low, while the EEG signal autocorrelation is high. CCA uses this property to remove the EMG artifacts which are mainly affected in the frequency range of $20 \mathrm{~Hz}-50 \mathrm{~Hz}$.

Consider that there are a multi-channel EEG signal $X(t)$ and its delayed version $Y(t)$ such that $Y(t)=X(t-1)$. The basis vectors are $x=w^{T} X$ and $y=w^{T} Y$. In this scenario, CCA maximizes the correlation between $x$ and $y$ by removing the mean of $x$ and $y$ [3].

$$
\rho(x, y)=\max _{w_{x}, w_{y}} \frac{E\left[X X^{T}\right]}{\left.\sqrt{E[X} X X^{T}\right] E\left[Y Y^{T}\right]}=\max _{w_{x}, w_{y}} \frac{E\left[w_{x}^{T} w_{y}^{T}\right]}{\sqrt{E\left[w_{x}^{T} w_{x}^{T}\right] E\left[w_{x}^{T} w_{y}^{T}\right]}}=\max \frac{w_{x}^{T} C_{x y} w}{\left(w_{x}^{T} C_{x x} w\right)\left(w_{y}^{T} C_{y y} w_{y}\right)}
$$

where $C_{x x}$ and $C_{y y}$ are auto-covariance matrices of $X$ and $Y$, respectively. $C_{x y}$ is the cross-covariance matrix of $X$ and $Y$. By calculating the derivative of Eq. (10) with respect to $w_{x}$ and $w_{y}$ and equating to zero, the following equations are derived.

$$
\begin{aligned}
& C_{x x}^{-1} C_{x y} C_{y y}^{-1} C_{y x} \hat{w_{x}}=\rho^{2} \hat{w_{x}} \\
& C_{y y}^{-1} C_{y x} C_{x x}^{-1} C_{x y} \hat{w_{y}}=\rho^{2} \hat{w_{y}}
\end{aligned}
$$

where $\rho$ is the canonical CC. $\rho$ is maximized such that artifact components can be removed. If $\rho$ is minimized, then these components approach to artifactual features.

\subsection{Wavelet transform $(W T)$}

The observed EEG signals are not entire because physiological and non-physiological signals contaminate the observed signals. For the better analysis of EEG signals, artifact signals are removed. In PCA and ICA, the drawback is maintaining orthogonality and large data requirements. These drawbacks are overcome by WT. WT decomposes the time-domain EEG signals by convolving a scaled and translated wavelet function [44].

$$
\psi_{a, b}(n)=\frac{1}{\sqrt{a}} \psi\left(\frac{n-b}{a}\right)
$$

where $a$ and $b$ are real numbers. $N=1,2,3, \ldots, N$. DWT removes OAs because the wavelets have resembled OAs [45]. Here, some of the basic wavelets are shown in Fig. 8. 


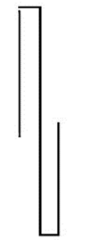

(a) Haar

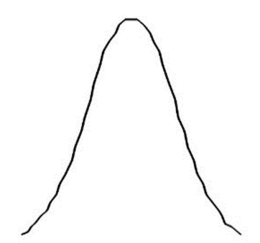

(e) Gaussian or spline

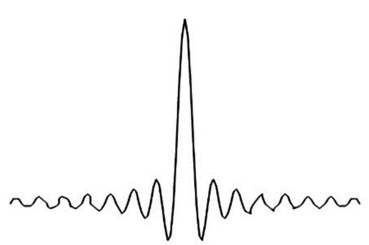

(b) Shannon or sinc

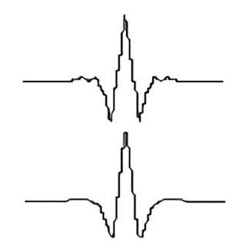

(f) Biorthogonal

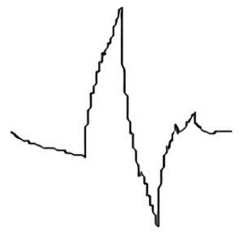

(c) Daubechies 4

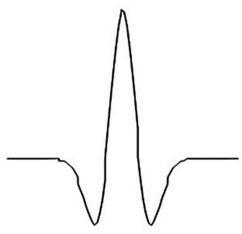

(g) Mexican hat

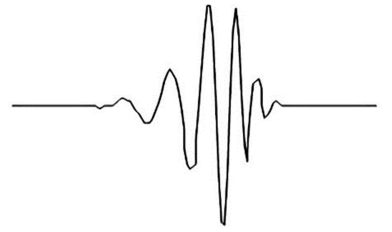

(d) Daubechies 20

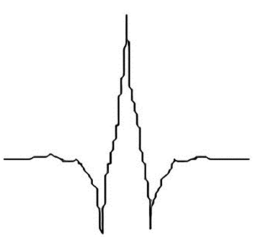

(h) Coiflet

Fig. 8 Graphical representation of different basic wavelets

DWT is a non-redundant and outstanding transform technique that aims to remove the artifacts from the time domain EEG signals. In this analysis, the signal is passed through a series of low-pass and high-pass filters to attain approximate and detailed coefficients [44]. The process is iteratively repeated until the desired frequency is reached. When this is achieved, the output is down-sampled by a factor of 2 and up-sampled to get the reconstructed signal. The basic block diagram of DWT is shown in Fig. 9.

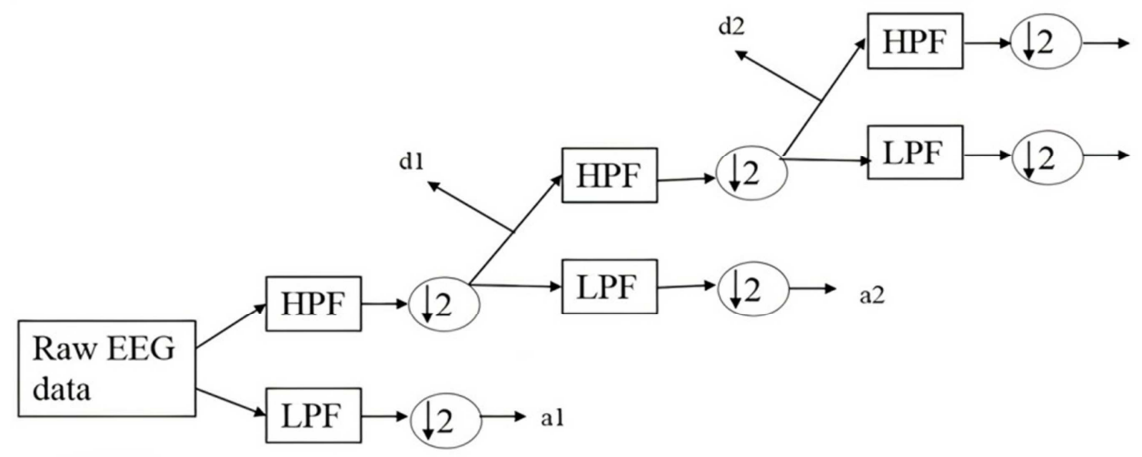

Fig. 9 DWT graphical decomposition representation [44]

$(\mathrm{HPF}=$ high-pass filter; $\mathrm{LPF}=$ low-pass filter $)$

DWT's advantage is that it can provide good temporal resolution for high frequency components and better frequency resolution for lower frequency components [19]. The major drawback of DWT is its time invariance. However, EEG data is not time-invariant and is randomly varying. This problem can be overcome by SWT, but the drawback is redundant information and slowness. In MATLAB, it is implemented by SWT function [46].

\subsection{Empirical mode decomposition (EMD)}

The practical recorded EEG signals are random stochastic signals, but not static signals. In the standard methods of artifact removal, the EEG signals are projected onto the basis vectors. In WT, the basis vectors are predefined. The experimentally recorded EEG data is not following the same pattern for different patients and, at other conditions, follows the random nature. Using predefined basis vectors to remove artifacts is not perfect because some proper EEG signals are also removed. A new method is proposed in 1998 to process nonlinear stochastic random data to overcome this problem [34]. EMD decomposes the EEG signals into some oscillatory functions called IMFs [47]. The derived IMFs must follow two criteria.

(1) In the whole dataset, the number of extremes and the number of zero-crossings must be equal or differ by at most one.

(2) At any point, the mean value of the envelope is given by the local maxima, and the minima must be zero. 
It is a data-driven method; unlike WT, the decomposition is not done by using predefined bass functions. Here are the steps for performing EMD [48]:

(1) For the given EEG signal $\mathrm{x}(\mathrm{t})$, identify all the local maxima and minima of the given signal.

(2) Interpolate between the maxima and minima to calculate the upper envelope $u(t)$ and lower envelope $1(\mathrm{t})$, respectively.

(3) Calculate the average $\mathrm{m} 1$ from the lower and upper envelopes and subtract it from the signal $\mathrm{x}(\mathrm{t})$ to get the first IMF.

$$
\begin{aligned}
& m_{1}=\frac{u(t)+l(t)}{2} \\
& h_{1}=x(t)-m_{1}
\end{aligned}
$$

(4) Check if $h_{1}$ satisfies the two criteria for IMF. If not, repeat the steps (1)-(3). After $i^{\text {th }}$ iteration, the conditions are satisfied.

$$
h_{1(i-1)}-m_{1 i}=h_{1 i}
$$

Then, $c_{1}=h_{1 i}$ becomes the first IMF. The stopping criteria are explained by Huang et al. [49-50], which are given by Cauchy convergence test. The first IMF is expected to have a high frequency.

$$
x(t)-c_{1}=r_{1}
$$

where $r_{1}$ is the first remainder, which contains remaining frequencies. Now, $r_{1}$ is the input signal for the next sifting process. The second IMF is $c_{2}$.

$$
\begin{aligned}
& r_{1}-c_{2}=r_{2} \\
& r_{n-1}-c_{n}=r_{n}
\end{aligned}
$$

This procedure is stopped when the residue is not satisfied with the IMF criteria and is a monotonic function. The given EEG signal is represented as:

$$
x(t)=\sum_{i=1}^{I}\left[c_{i}(t)+r_{I}(t)\right]
$$

EMD decomposes the signals based on amplitude and frequency information. The EMG artifacts cannot be separated from the EEG signals due to the overlapping at higher frequencies, but these kinds of EMG artifacts can be removed using some standard forms of recent artifact removal techniques.

\subsection{Singular spectrum analysis (SSA)}

SSA is a powerful decomposition method for the removal of artifacts in single-channel and multi-channel EEG signals. The BSS method is mainly applied to multi-channel data. In SSA, the procedure is primarily divided into two steps, and each step is further divided into two phases [51].

(1) Decomposition: (i) time delay embedding; (ii) SVD.

(2) Reconstruction: (i) grouping; (ii) diagonal averaging. 
Consider that there are $N$ sampled signals $x(t)=x_{1}, x_{2}, \ldots, x_{\mathrm{N}}$, then in the first embedding step, the $x(t)$ is mapped as $X$ whose size is $K \times P[52]$.

$$
X=\left[x_{1}, x_{2}, \cdots, x_{N}=\left(\begin{array}{cccc}
x_{1} & x_{2} & \cdots & x_{p} \\
x_{2} & x_{3} & \cdots & x_{p+1} \\
\vdots & \vdots & \ddots & \vdots \\
x_{L} & x_{L+1} & \cdots & x_{N}
\end{array}\right)\right]
$$

where $L$ is the embedding size. The general value $L=N / 2 . X_{i}$ is a lagged vector. The second step is SVD. By using SVD, the trajectory matrix $X$ is defined as:

$$
X_{i}=\sqrt{\lambda_{i}} V_{i} U_{i}
$$

where $\lambda_{i}$ is eigenvalue; $U_{i}$ and $V_{i}$ are left and right singular eigenvectors. Now the trajectory matrix $X$ is written as:

$$
X=\sum_{i=1}^{L} X_{i}
$$

As the indices $I_{n}$ are divided into $R$ groups. The matrix $X$ after grouping is:

$$
X=\sum_{j=1}^{R} X_{I_{j}}
$$

In the last step of diagonal averaging, the sub-matrix $X_{I_{j}}$ is translated to new series by the averaging of anti-diagonal elements. The final trajectory matrix is represented as:

$$
x(t)=\sum_{j=1}^{L} x(t)_{j}^{r c}
$$

The SSA method can remove muscle artifacts better compared to BSS methods. By combing the SSA with other BSS methods, artifacts can be removed further.

\subsection{Independent vector analysis (IVA)}

The EEG data is contaminated by noises/artifacts while recording from the scalp. There are many methods for removing EOG artifacts, e.g., ICA, CCA, EMD, SSA, etc. The removal of muscle artifacts is difficult; only a few methods are there because muscle artifacts have high amplitude and variable topographical distribution, and overlap with the entire EEG spectrum [53]. ICA separates the muscle artifacts effectively using HOS by linearly un-mixing the EEG data into ICs if and only if the artifacts are stereotyped. However, muscle artifacts are not stereotyped because these are generated from a group of muscles. CCA extracts the sources maximally autocorrelated and mutually uncorrelated using SOS. Comparing with EEG signals, muscle artifacts have a low correlation. CCA uses this property to remove the artifacts. CCA and ICA are applicable mainly for multi-channel data. Kim et al. [54] have proposed an IVA approach similar to ICA, and have addressed the permutation issue when dealing with acoustic source separation. In recent times, this approach has been applied to EEG signals and could remove muscle artifacts successfully.

IVA operation which relies on "source extraction from different datasets and maximally dependent sources" within the dataset is maximally independent [53]. In this analysis, the source independence within the dataset and dependence between the datasets exist in parallel. In IVA, the source component vector (SCV) was defined from different datasets [54]. 
The $p^{\text {th }} \mathrm{SCV}$ is $s_{p}=\left[s_{p}^{1}, s_{p}^{2}, \ldots, s_{p}^{M}\right](p=1,2, \ldots, P)$, which is a random vector independent of all other SCVs. $s_{p}^{m}(m=1$, $2, \ldots, M)$ represents the $p^{\text {th }}$ source component in the $m^{\text {th }}$ dataset. The main intention of IVA approach is to identify the independent SCV from the multi-dimensional dataset, which is achieved by minimizing the MI among the SCV estimates $\hat{s}_{p}$.

$$
I_{I V A} \triangleq I\left[\hat{s}_{1} ; \hat{s}_{2} ; \ldots \ldots \hat{s}_{p}\right]
$$

where $I\left[\hat{s}_{p}\right]$ is the MI within the $p^{\text {th }} \mathrm{SCV}$.

Initially, IVA assumes that the data follow Laplacian distribution (IVA-L), which uses HOS without considering linear dependence using SOS. Anderson et al. [53] proposed IVA-G with the Gaussian distribution model, which uses SOS and does not consider linear dependence. Recently, Chen et al. [55] in 2017 implemented a novel IVA algorithm with multivariate power exponential distribution (MPE), which uses both SOS and HOS to get both advantages like linear independence and correlation. The drawback of IVA is computational complexity and simulation time. It is mainly applied in laboratory applications, not in online applications.

\subsection{Hybrid methods}

Artifact removal in EEG signals is vital for analyzing brain conditions and diagnosing the patient if any abnormalities are there. Researchers are trying some new techniques by combing more than one existing previously discussed method for better removal of artifacts. By combining one or more methods, the advantages of each technique can be used to develop a new modality for completely removing the artifacts. A hybrid method utilizes the combination of several filters to eliminate the artifacts. It can eliminate the significant artifacts from the EEG signals without adjusting the interpretation of the neural state in the real and simulated EEG data. It improves the previous versions of total variation denoising and EMD variants.

\subsubsection{Discrete wavelet transform - adaptive filtering method (DWT-AFM)}

For removing the artifacts in EEG signals using ICA, a reference signal is required. This problem can be resolved by using DWT. The reference EOG signal can be generated from the given data by selecting low-frequency wavelet coefficients to remove OAs [56]. Unlike ICA, a large amount of information is also not required for better removal of artifacts. However, the disadvantage of DWT is that it cannot remove the artifacts effectively if the spectrum of artifacts overlap with the original EEG signals [44]. The AFM is also called adaptive noise canceler (ANC). ANC can solve this problem. The drawback of ANC is that it requires a reference signal for the removal of artifacts. By combing DWT and ANC, both spectral overlap and reference signal problems can be solved [33]. For OA removal, DWT is used for generating reference EOG signal, and this reference signal is used as an input reference for ANC. With this hybrid method, the removal of artifacts is better compared to using DWT and ANC independently.

\subsubsection{Discrete wavelet transform - blind source separation (DWT-BSS)}

DWT-BSS is one of the hybrid methods to remove the artifacts more effectively. The BSS technique is generally applicable to multi-channel data. The disadvantages of both methods are discussed in previous sections. To overcome these disadvantages by combing these methods, the advantages in both methods can be combined. Based on single-channel and multi-channel, one of these methods can be used as the first method to apply. First, DWT is applied to decompose the time series signal into wavelet coefficients for the single-channel technique, and these coefficients are applied to BSS (ICA or CCA) [46]. For multi-channel data, DWT or BSS can be used first, and the resultant signal is applied to the second method for the removal of artifacts. If ICA and CCA are applied first, the time domain signal is decomposed into ICs and CCs, and by using DWT, these coefficients are further decomposed to better remove the artifacts from the EEG data [19]. 


\subsubsection{Empirical mode decomposition - blind source separation (EMD-BSS)}

BSS techniques are used for decomposing multi-channel data into separate ICs and CCs using ICA and CCA, respectively. These techniques are mainly helpful for laboratory experiments, where multi-channel data is available. However, for ambulatory or single-channel data analysis, different methods should be used, e.g., DWT, EMD, SSA, etc. The performance can be increased in terms of artifact removal using a hybrid model combing multiple methods. DWT decomposes the data using predefined mother wavelets [57]. However, for nonlinear and non-stationary stochastic data like EEG signals, DWT is not sufficient. EMD is a data-driven technique that effectively decomposes randomly varying data like single-channel EEG signals into IMFs [10]. Then, by using any BSS like ICA or CCA, these IMFs can be further decomposed to remove artifacts. For multi-channel data, EMD can also apply channel-wise to decompose into IMFs [43].

\subsubsection{Singular spectrum analysis - adaptive noise canceler (SSA-ANC)}

SSA-ANC is one of the hybrid methods for the removal of artifacts from single-channel and multi-channel EEG signals. It can be applied to portable devices, where multi-channel data collection is not possible because ICA and CCA are multi-channel data methods. In the ANC method for EOG artifact removal, a reference EOG artifactual signal is required. In a hybrid method like SSA-ANC, the reference signal for removing EOG artifacts for ANC is given by SSA, like DWT, based on eigenvalues. In DWT, last few low-frequency components are used for generating the EOG reference signal [56]. In SSA, the reference signal is also generated from the lower eigenvalues, which are developed while applying SVD for the trajectory matrix [58]. The performance is improved in terms of artifact removal compared with SSA and ANC because the input reference EOG signal is given for ANC, which is generated from the output of SSA.

\subsubsection{Singular spectrum analysis - blind source separation (SSA-BSS)}

ICA and CCA are powerful BSS methods that use SOS and HOS to remove OAs and muscle artifacts from the recorded EEG signals [57]. BSS methods are multi-channel techniques, and each one has its own advantages and disadvantages. BSS methods are combined with SSA as a hybrid method to remove artifacts from the recorded EEG signals. SSA decomposes the single-channel or multi-channel EEG data, and the decomposed data is further processed by BSS methods to remove artifacts.

\subsubsection{Empirical mode decomposition - independent vector analysis (EMD-IVA)}

IVA uses SOS and HOS for the removal of artifacts [55]. By combing with EMD, IVA removes the artifacts effectively compared with IVA independently. EMD method decomposes the single-channel signals into multiple IMFs, and these IMFs are applied to IVA to remove artifacts effectively. To overcome the disadvantages in EMD, noise-assisted EMD is proposed, which is EEMD. EEMD is applied to single-channel, but MEMD is applied to multi-channel data also. By combining MEMD and IVA, Zou et al. [12] proposed a method MEMD-IVA for removing the artifacts from multi-channel signals.

\section{Comparative Analysis of the Existing Methods}

A tabulated survey of literature and methods are presented in Table 4. No independent method can remove all the artifacts. The categorization of procedures is done based on the reference channel requirement. The automatic process can be applied online, whether single-channel or multi-channel, but prior information is required to improve the performance to remove artifacts. Some methods are used for physiological artifact removal, and some are for non-physiological artifact removal. Regression methods are elementary for the removal of artifacts but require reference channels and multi-channel data. Filtering methods remove the artifacts by first estimating the artifactual components and subtracting them from the original EEG contaminated signals, but part of the EEG signals is also removed while subtracting. In KF, the first artifactual region is 
identified, and by estimating noise, it will be subtracted from only the contaminated area [35]. BSS methods are very effective and helpful for the removal of artifacts. Most researchers use ICA to remove artifacts but are restricted to stereotyped artifacts only [61]. Muscle artifacts are not stereotyped and thus cannot be removed by ICA effectively. CCA can overcome this problem, but it increases computational cost and simulation time, making CCA not being applied in online applications. ICA and CCA are multi-channel data processing methods that cannot be used for ambulatory analysis where few channels or single-channel data is available.

Table 4 Comparative analysis of artifactual methods discussed in the survey

\begin{tabular}{|c|c|c|c|c|c|c|}
\hline Method & $\begin{array}{c}\text { Reference } \\
\text { electrode required }\end{array}$ & $\begin{array}{c}\text { Prior } \\
\text { knowledge }\end{array}$ & Automatic & Online & $\begin{array}{c}\text { Single-/ } \\
\text { multi-channel }\end{array}$ & Ref. \\
\hline Regression & Yes & No & Yes & No & Multi & {$[33]$} \\
\hline AF & Yes & Yes & Yes & Yes & Both & {$[34]$} \\
\hline KF & No & Yes & Yes & Yes & Both & {$[35]$} \\
\hline PCA & No & No & No & No & Multi & {$[8]$} \\
\hline ICA & No & No & Yes & Yes & Multi & {$[2]$} \\
\hline CCA & No & No & Yes & Yes & Multi & {$[3]$} \\
\hline DWT & No & No & No & No & Both & {$[44]$} \\
\hline EMD & No & No & No & Yes & Both & {$[48]$} \\
\hline SSA & No & No & Yes & No & Both & {$[52]$} \\
\hline IVA & No & No & No & No & Both & {$[54]$} \\
\hline DWT-AFM & No & No & Yes & Yes & Both & {$[56]$} \\
\hline DWT-BSS & No & No & No & No & Both & {$[46]$} \\
\hline EMD-BSS & No & No & No & No & Both & {$[10,59]$} \\
\hline SSA-ANC & No & No & Yes & Yes & Both & {$[57]$} \\
\hline SSA-BSS & No & No & No & No & Both & {$[57]$} \\
\hline EMD-IVA & No & No & No & No & Both & {$[55,60]$} \\
\hline
\end{tabular}

EMD and SSA methods can be applied for both single-channel and multi-channel, but the disadvantage is mode mixing and aliasing in EMD. SSA method removes only one type of artifacts. An extension of ICA from 1-D data is IVA technology, which can be used to remove both ocular and muscle artifacts as it takes advantage of both CCA and ICA methods. Automatic methods can remove artifacts without manual intervention. Regression methods can be used as intuitive if the reference channel is there [8]. BSS methods are used as automatic if a subsequent procedure like SVM is applied to verify artifactual components.

Individually each method has its advantages and disadvantages. Researchers are combing more than one method like a hybrid method to get better results to use the benefits of both approaches. In hybrids methods, multi-channel strategies can be applied for single-channels by applying single-channel techniques first. The output is applied as the input for the multi-channel approach. EEMD-ICA method removes the EOG artifacts, and EEMD-CCA removes EMG artifacts from a single-channel, even though ICA and CCA are multi-channel methods [62]. EEMD decomposes the single-channel data into multiple IMFs. These IMFs can be applied to ICA or CCA for further processing to remove artifacts. Compared to WT-ICA, EEMD-ICA removes artifacts effectively because EEMD is a data-driven method. FMEMD-CCA is used to remove muscle artifacts from a few channel methods with less computational time [25]. WT-ICA combined with SVM was proposed to remove OAs automatically. The recursive least square (RLS) notch filter method is proposed to remove ECG artifacts because regression and filtering methods cannot delete the ECG artifacts [63]. The MEMD-IVA method is proposed to remove the muscle artifacts from the acquisition channels, in which the inter-channel interference is also considered one of the noises [60]. Some recommendations are suggested to remove muscle artifacts from a few channel data [64]. All the above methods remove the artifacts effectively by hybrid application. Apart from these methods, researchers will propose one optimal method to remove all the artifacts in the future. 


\section{Conclusions}

EEG signals are generated from the brain and recorded from the scalp using electrodes. Different physiological and non-physiological artifacts contaminate these time-domain EEG signals. In this study, many methods are summarized from various author's publications, but no specific method can eradicate the artifacts with high accuracy and efficiency. The advantages and disadvantages of each method are discussed in this study. Some methods are used primarily to remove artifacts such as EOG, EMG, and ECG, but no specific method can remove all the artifacts. Regression methods and filtering methods require artifactual reference signals for the removal of artifacts. BSS methods remove the artifacts effectively, but they require multi-channel extensive data and are computationally complex. DWT, EMD, and SSA methods are used for single-channel and multi-channel, but the efficiency of removing artifacts is not up to the level. By combining more than one method, i.e., the hybrid method, the efficiency is improved compared to individual methods. Still, cost and complexity is increased, and this may not be suitable for online applications. In this aspect, researchers are finding an optimal solution for minimizing the artifacts in the EEG signals. In the future, with the existing artifact removal techniques, one specific method will be implemented by combing present trends, e.g., machine learning, artificial intelligence (AI), and genetic algorithm (GA), with increased accuracy and efficiency for the removal of artifacts.

\section{Nomenclature}

\begin{tabular}{|c|c|c|c|}
\hline EEG & Electroencephalogram & MAX & Maximum absolute error \\
\hline BSS & Blind source separation & NRD & Normalized root mean square difference \\
\hline $\mathrm{OA}$ & Ocular artifacts & PRD & Percentage root mean square difference \\
\hline EMG & Electromyography & NMSE & Normalized mean square error \\
\hline ECG & Electrocardiography & PSNR & Peak signal to noise ratio \\
\hline mMSE & modified multiscale sample entropy & FAR & False alarm rate \\
\hline IVA & Independent vector analysis & MEV & Mean entropy value \\
\hline WT & Wavelet transform & DSNR & Difference signal to noise ratio \\
\hline SWT & Stationary wavelet transform & $\mathrm{AF}$ & Adaptive filtering \\
\hline DWT & Discrete wavelet transform & EOG & Electrooculogram \\
\hline ST & Statistical threshold & EKF & Extension Kalman filter \\
\hline $\mathrm{UT}$ & Universal threshold & UKF & Unscented Kalman filter \\
\hline EM & Eye movement & PCA & Principle component analysis \\
\hline ANN & Artificial neural network & $\mathrm{PC}$ & Principle component \\
\hline DOA & Depth of anaesthesia & SVD & Singular value decomposition \\
\hline BIS & Bispectral index & $\mathrm{CCA}$ & Canonical correlation analysis \\
\hline ARTE & Automated artifact handling in EEG & ANC & Adaptive noise canceler \\
\hline SVM & Support vector machine & $\mathrm{CC}$ & Correlation coefficient \\
\hline ASR & Artifact subspace reconstruction & MI & Mutual information \\
\hline EEMD & Enhanced empirical mode decomposition & SOS & Second-order statistics \\
\hline SQI & Signal quality index & HOS & Higher-order statistics \\
\hline IMF & Intrinsic mode function & AI & Artificial intelligence \\
\hline MEMD & Multivariate empirical mode decomposition & GA & Genetic algorithm \\
\hline FMEMD & Fast multivariate empirical mode decomposition & SNR & Signal to noise ratio \\
\hline BLIMF & Band limited intrinsic mode function & MSE & Mean square error \\
\hline SSA & Singular spectrum analysis & MAE & Mean absolute error \\
\hline NNR & Neural network regressor & $\mathrm{RE}$ & Relative error \\
\hline MRAF & Multi-resolutional analysis and adaptive filtering & RMSE & Root mean square error \\
\hline WPD & Wavelet packet decomposition & RRMSE & Relative root mean square error \\
\hline ICA & Independent component analysis & NRMSE & Normalized root mean square error \\
\hline $\mathrm{IC}$ & Independent component & PSD & Power spectral density \\
\hline TUH & Temple university hospital & SAR & Signal to artifact ratio \\
\hline
\end{tabular}




\section{Conflicts of Interest}

The authors declare no conflict of interest.

\section{Statement of Ethical Approval}

(a) Statement of human rights

For this type of study, statement of human rights is not required.

(b) Statement on the welfare of animals

For this type of study, statement on the welfare of animals is not required.

\section{Statement of Informed Consent}

For this type of study, informed consent is not required.

\section{References}

[1] H. Hu, S. Guo, R. Liu, and P. Wang, “An Adaptive Singular Spectrum Analysis Method for Extracting Brain Rhythms of Electroencephalography,” Peerj, vol. 5, e3474, June 2017.

[2] A. K. Abdullah, C. Z. Zhang, A. A. A. Abdullah, and S. Lian, "Automatic Extraction System for Common Artifacts in EEG Signals Based on Evolutionary Stone's BSS Algorithm,” Mathematical Problems in Engineering, vol. 2014, 324750, August 2014.

[3] M. M. N. Mannan, M. A. Kamran, and M. Y. Jeong, "Identification and Removal of Physiological Artifacts from Electroencephalogram Signals: A Review,” IEEE Access, vol. 6, pp. 30630-30652, 2018.

[4] R. Zafar, A. Qayyum, and W. Mumtaz, "Automatic Eye Blink Artifact Removal for EEG Based on a Sparse Coding Technique for Assessing Major Mental Disorders,” Journal of Integrative Neuroscience, vol. 18, no. 3, pp. $217-229,2019$.

[5] A. Morley, L. Hill, and A. G. Kaditis, "10-20 System EEG Placement," https://www.Ers-Education.Org/Lrmedia/2016/Pdf/298830.Pdf, March 15, 2016.

[6] S. K. Goh, H. A. Abbass, K. C. Tan, A. Al-Mamun, C. Wang, and C. Guan, "Automatic EEG Artifact Removal Techniques by Detecting Influential Independent Components," IEEE Transactions on Emerging Topics in Computational Intelligence, vol. 1, no. 4, pp. 270-279, August 2017.

[7] S. R. Sreeja, R. R. Sahay, D. Samanta, and P. Mitra, "Removal of Eye Blink Artifacts from EEG Signals Using Sparsity," IEEE Journal of Biomedical and Health Informatics, vol. 22, no. 5, pp. 1362-1372, September 2018.

[8] X. Jiang, G. B. Bian, and Z. Tian, "Removal of Artifacts from EEG Signals: A Review," Sensors, vol. 19, no. 5, 987, March 2019.

[9] S. S. Menon and K. Krishnamurthy, "A Study of Brain Neuronal and Functional Complexities Estimated Using Multiscale Entropy in Healthy Young Adults,” Entropy, vol. 21, no. 10, 995, October 2019.

[10] K. T. Sweeney, S. F. McLoone, and T. E. Ward, "The Use of Ensemble Empirical Mode Decomposition with Canonical Correlation Analysis as a Novel Artifact Removal Technique,” IEEE Transactions on Biomedical Engineering, vol. 60, no. 1, pp. 97-105, January 2013.

[11] M. Chavez, F. Grosselin, A. Bussalb, F. D. V. Fallani, and X. Navarro-Sune, "Surrogate-Based Artifact Removal from Single-Channel EEG,” IEEE Transactions on Neural Systems and Rehabilitation Engineering, vol. 26, no. 3, pp. 540-550, March 2018.

[12] Y. Zou, V. Nathan, and R. Jafari, “Automatic Identification of Artifact-Related Independent Components for Artifact Removal in EEG Recordings,” IEEE Journal of Biomedical and Health Informatics, vol. 20. no. 1, pp. 73-81, January 2016.

[13] R. Mahajan and B. I. Morshed, "Unsupervised Eye Blink Artifact Denoising of EEG Data with Modified Multiscale Sample Entropy, Kurtosis, and Wavelet-ICA,” IEEE Journal of Biomedical and Health Informatics, vol. 19, no. 1, pp. 158-165, January 2015. 
[14] P. P. Acharjee, R. Phlypo, L. Wu, V. D. Calhoun, and T. Adal1, "Independent Vector Analysis for Gradient Artifact Removal in Concurrent EEG-fMRI Data," IEEE Transactions on Biomedical Engineering, vol. 62, no. 7, pp. 1750-1758, July 2015.

[15] S. Khatun, R. Mahajan, and B. I. Morshed, "Comparative Study of Wavelet-Based Unsupervised Ocular Artifact Removal Techniques for Single-Channel EEG Data,” IEEE Journal of Translational Engineering in Health and Medicine, vol. 4, pp. 1-8, March 2016.

[16] M. N. Tibdewal, R. R. Fate, M. Mahadevappa, A. K. Ray, and M. Malokar, "Classification of Artifactual EEG Signal and Detection of Multiple Eye Movement Artifact Zones Using Novel Time-Amplitude Algorithm," Signal, Image, and Video Processing, vol. 11, no. 2, pp. 333-340, February 2017.

[17] Q. Liu, Y. F. Chen, S. Z. Fan, M. F. Abbod, and J. S. Shieh, "EEG Artifacts Reduction by Multivariate Empirical Mode Decomposition and Multiscale Entropy for Monitoring Depth of Anaesthesia During Surgery," Medical and Biological Engineering and Computing, vol. 55, no. 8, pp. 1435-1450, August 2017.

[18] S. Barua, M. U. Ahmed, C. Ahlstrom, S. Begum, and P. Funk, “Automated EEG Artifact Handling with Application in Driver Monitoring," IEEE Journal of Biomedical and Health Informatics, vol. 22, no. 5, pp. 1350-1361, September 2018.

[19] C. Y. Sai, N. Mokhtar, H. Arof, P. Cumming, and M. Iwahashi, "Automated Classification and Removal of EEG Artifacts with SVM and Wavelet-ICA,” IEEE Journal of Biomedical and Health Informatics, vol. 22, no. 3, pp. 664-670, May 2018.

[20] J. Li, Y. Chen, F. Taya, J. Lim, K. Wong, Y. Sun, et al., “A Unified Canonical Correlation Analysis-Based Framework for Removing Gradient Artifact in Concurrent EEG/fMRI Recording and Motion Artifact in Walking Recording from EEG Signal," Medical and Biological Engineering and Computing, vol. 55, no. 9, pp. 1669-1681, September 2017.

[21] V. Roy and S. Shukla, "Effective EEG Motion Artifacts Elimination Based on Comparative Interpolation Analysis," Wireless Personal Communications, vol. 97, no. 4, pp. 6441-6451, December 2017.

[22] C. Y. Chang, S. H. Hsu, L. Pion-Tonachini, and T. P. Jung, "Evaluation of Artifact Subspace Reconstruction for Automatic Artifact Components Removal in Multi-Channel EEG Recordings," IEEE Transactions on Biomedical Engineering, vol. 67, no. 4, pp. 1114-1121, April 2020.

[23] J. Cheng, L. Li, C. Li, Y. Liu, A. Liu, R. Qian, et al., "Remove Diverse Artifacts Simultaneously from a Single-Channel EEG Based on SSA and ICA: A Semi-Simulated Study,” IEEE Access, vol. 7, pp. 60276-60289, 2019.

[24] M. Saini and U. Satija, "An Effective and Robust Framework for Ocular Artifact Removal from Single-Channel EEG Signal Based on Variational Mode Decomposition,” IEEE Sensors Journal, vol. 20, no. 1, pp. 369-376, January 2020.

[25] Y. Liu, Y. Zhou, X. Lang, Y. Liu, Q. Zheng, Y. Zhang, et al., “An Efficient and Robust Muscle Artifact Removal Method for Few-Channel EEG,” IEEE Access, vol. 7, pp. 176036-176050, 2019.

[26] C. Dora and P. K. Biswal, "Correlation-Based ECG Artifact Correction from Single Channel EEG Using Modified Variational Mode Decomposition,” Computer Methods and Programs in Biomedicine, vol. 183, 105092, January 2020.

[27] C. Dora, R. N. Patro, S. K. Rout, P. K. Biswal, and B. Biswal, “Adaptive SSA Based Muscle Artifact Removal from Single Channel EEG Using Neural Network Regressor," Innovation and Research in Biomedical Engineering, in press.

[28] D. P. Yedurkar and S. P. Metkar, "Multiresolution Approach for Artifacts Removal and Localization of Seizure onset Zone in Epileptic EEG Signal,” Biomedical Signal Processing and Control, vol. 57, 101794, March 2020.

[29] M. S. Islam, A. M. El-Hajj, H. Alawieh, Z. Dawy, N. Abbas, and J. El-Imad, "EEG Mobility Artifact Removal for Ambulatory Epileptic Seizure Prediction Applications,” Biomedical Signal Processing and Control, vol. 55, 101638, January 2020.

[30] N. Bajaj, J. R. Carrión, F. Bellotti, R. Berta, and A. De Gloria, “Automatic and Tunable Algorithm for EEG Artifact Removal Using Wavelet Decomposition with Applications in Predictive Modeling During Auditory Tasks," Biomedical Signal Processing and Control, vol. 55, 101624, January 2020.

[31] S. K. Noorbasha and G. F. Sudha, "Removal of EOG Artifacts and Separation of Different Cerebral Activity Components from Single Channel EEG-An Efficient Approach Combining SSA-ICA with Wavelet Thresholding for BCI Applications,” Biomedical Signal Processing and Control, vol. 63, 102168, January 2021.

[32] M. A. Klados, C. Papadelis, C. D. Lithari, and P. D. Bamidis, "The Removal of Ocular Artifacts from EEG Signals: A Comparison of Performances for Different Methods," 4th European Conference of the International Federation for Medical and Biological Engineering, pp. 1259-1263, November 2008.

[33] Y. Chen, Q. Zhao, B. Hu, J. Li, H. Jiang, W. Lin, et al., “A Method of Removing Ocular Artifacts from EEG Using Discrete Wavelet Transform and Kalman Filtering," IEEE International Conference on Bioinformatics and Biomedicine, pp. 15-18, December 2016.

[34] A. Zhang and W. Li, "Adaptive Noise Cancellation for Removing Cardiac and Respiratory Artifacts from EEG Recordings," 5th World Congress on Intelligent Control and Automation, pp. 15-19, June 2004. 
[35] H. Shahabi, S. Moghimi, and H. Zamiri-Jafarian, "EEG Eye Blink Artifact Removal by EOG Modeling and Kalman Filter," 5th International Conference on Biomedical Engineering and Informatics, pp. 356-367, October 2012.

[36] A. Mur, R. Dormido, and N. Duro, “An Unsupervised Method for Artefact Removal in EEG Signals,” Sensors, vol. 19, no. 10, 2302, May 2019.

[37] B. Mijović, M. De Vos, I. Gligorijević, J. Taelman, and S. Van Huffel, "Source Separation from Single-Channel Recordings by Combining Empirical-Mode Decomposition and Independent Component Analysis," IEEE Transactions on Biomedical Engineering, vol. 57, no. 9, pp. 2188-2196, September 2010.

[38] V. Roy and S. Shukla, "A Survey on Artifacts Detection Techniques for Electro-Encephalography (EEG) Signals," International Journal of Multimedia and Ubiquitous Engineering, vol. 10, no. 3, pp. 425-442, 2015.

[39] G. Wang, C. Teng, K. Li, Z. Zhang, and X. Yan, "The Removal of EOG Artifacts from EEG Signals Using Independent Component Analysis and Multivariate Empirical Mode Decomposition," IEEE Journal of Biomedical and Health Informatics, vol. 20, no. 5, pp. 1301-1308, September 2016.

[40] X. Chen, A. Liu, H. Peng, and R. K. Ward, “A Preliminary Study of Muscular Artifact Cancellation in Single-Channel EEG,” Sensors, vol. 14, no. 10, pp. 18370-18389, October 2014.

[41] D. R. Hardoon, S. Szedmak, and J. Shawe-Taylor, "Canonical Correlation Analysis: An Overview with Application to Learning Methods,” Neural Computation, vol. 16, no. 12, pp. 2639-2664, December 2004.

[42] W. De Clercq, A. Vergult, B. Vanrumste, W. Van Paesschen, and S. Van Huffel, "Canonical Correlation Analysis Applied to Remove Muscle Artifacts from the Electroencephalogram,” IEEE Transactions on Biomedical Engineering, vol. 53, no. 12, pp. 2583-2587, 2006.

[43] X. Chen, X. Xu, A. Liu, M. J. Mckeown, and Z. J. Wang, "The Use of Multivariate EMD and CCA for Denoising Muscle Artifacts from Few-Channel EEG Recordings," IEEE Transactions on Instrumentation and Measurement, vol. 67, no. 2, pp. 359-370, February 2018.

[44] S. Khatun, R. Mahajan, and B. I. Morshed, "Comparative Study of Wavelet-Based Unsupervised Ocular Artifact Removal Techniques for Single Channel EEG Data," IEEE Journal of Translational Engineering in Health and Medicine, vol. 4, 2000108, 2016.

[45] P. Gajbhiye, R. K. Tripathy, A. Bhattacharyya, and R. B. Pachori, "Novel Approaches for the Removal of Motion Artifact from EEG Recordings,” IEEE Sensors Journal, vol. 19, no. 22, pp. 10600-10608, November 2019.

[46] B. S. Raghavendra and D. N. Dutt, "Wavelet Enhanced CCA for Minimization of Ocular and Muscle Artifacts in EEG," World Academy of Science, Engineering, and Technology, vol. 5, no. 9, pp. 419-424, January 2011.

[47] E. Karatoprak and S. Seker, "An Improved Empirical Mode Decomposition Method Using Variable Window Median Filter for Early Fault Detection in Electric Motors,” Mathematical Problems in Engineering, vol. 2019, 8015295, 2019.

[48] B. Yang, T. Zhang, Y. Zhang, W. Liu, J. Wang, and K. Duan, "Removal of Electrooculogram Artifacts from Electroencephalogram Using Canonical Correlation Analysis with Ensemble Empirical Mode Decomposition," Cognitive Computation, vol. 9, no. 5, pp. 626-633, October 2017.

[49] N. E. Huang and Z. Wu, "A Review on Hilbert-Huang Transform: Method and its Applications to Geophysical Studies," Reviews of Geophysics, vol. 46, no. 2, RG2006, June 2008.

[50] N. E. Huang, M. L. C. Wu, S. R. Long, S. S. Shen, W. Qu, P. Gloersen, et al., "A Confidence Limit for the Empirical Mode Decomposition and Hilbert Spectral Analysis," Proceedings of the Royal Society A: Mathematical, Physical, and Engineering Sciences, vol. 459, no. 2037. pp. 2317-2345, September 2003.

[51] S. Xu, H. Hu, L. Ji, and P. Wang, "Embedding Dimension Selection for Adaptive Singular Spectrum Analysis of EEG Signal," Sensors, vol. 18, no. 3, 697, March 2018.

[52] Q. Liu, A. Liu, X. Zhang, X. Chen, R. Qian, and X. Chen, "Removal of EMG Artifacts from Multichannel EEG Signals Using Combined Singular Spectrum Analysis and Canonical Correlation Analysis,” Journal of Healthcare Engineering, vol. 2019, 4159676, 2019.

[53] M. Anderson, T. Adali, and X. L. Li, "Joint Blind Source Separation with Multivariate Gaussian Model: Algorithms and Performance Analysis,” IEEE Transaction on Signal Process, vol. 60, no. 4, pp. 1672-1683, April 2012.

[54] T. Kim, T. Eltoft, and T. W. Lee, "Independent Vector Analysis: An Extension of ICA to Multivariate Components," International Conference on Independent Component Analysis and Signal Separation, March 2006, pp. $165-172$.

[55] X. Chen, A. Liu, Q. Chen, Y. Liu, L. Zuo, and M. J. Mckeown, "Simultaneous Ocular and Muscle Artifact Removal from EEG Data by Exploiting Diverse Statistics," Computers in Biology and Medicine, vol. 88, pp. 1-10, September 2017.

[56] H. Peng, B. Hu, Q. Shi, M. Ratcliffe, Q. Zhao, Y. Qi, et al., "Removal of Ocular Artifacts in EEG-An Improved Approach Combining DWT and ANC for Portable Applications," IEEE Journal of Biomedical and Health Informatics, vol. 17, no. 3, pp. 600-607, May 2013. 
[57] L. Zou, X. Chen, G. Dang, Y. Guo, and Z. J. Wang, "Removing Muscle Artifacts from EEG Data via Underdetermined Joint Blind Source Separation: A Simulation Study,” IEEE Transactions on Circuits and Systems II: Express Briefs, vol. 67, no. 1, pp. 187-191, January 2020.

[58] A. K. Maddirala and R. A. Shaik, "Removal of EOG Artifacts from Single Channel EEG Signals Using Combined Singular Spectrum Analysis and Adaptive Noise Canceler,” IEEE Sensors Journal, vol. 16, no. 23, pp. 8279-8287, December 2019.

[59] X. Chen, A. Liu, J. Chiang, Z. J. Wang, M. J. Mckeown, and R. K. Ward, "Removing Muscle Artifacts from EEG Data: Multi-Channel or Single-Channel Techniques,” IEEE Sensors Journal, vol. 16, no. 7, pp. 1986-1997, April 2016.

[60] X. Xu, X. Chen, and Y. Zhang, "Removal of Muscle Artefacts from Few-Channel EEG Recordings Based on Multivariate Empirical Mode Decomposition and Independent Vector Analysis,” Electronics Letters, vol. 54, no. 14, pp. 866-868, July 2018.

[61] X. Chen, Q. Liu, W. Tao, L. Li, S. Lee, A. Liu, et al., "ReMAE: User-Friendly Toolbox for Removing Muscle Artifacts from EEG,” IEEE Transactions on Instrumentation and Measurement, vol. 69, no. 5, pp. 2105-2119, May 2020.

[62] X. Chen, Q. Chen, Y. Zhang, and Z. J. Wang, “A Novel EEMD-CCA Approach to Removing Muscle Artifacts for Pervasive EEG,” IEEE Sensors Journal, vol. 19, no. 19. pp. 8420-8431, October 2019.

[63] C. Dai, J. Wang, J. Xie, W. Li, Y. Gong, and Y. Li, "Removal of ECG Artifacts from EEG Using an Effective Recursive Least Square Notch Filter," IEEE Access, vol. 7, pp. 158872-158880, October 2019.

[64] X. Chen, X. Xu, A. Liu, S. Lee, X. Chen, X. Zhang, et al., "Removal of Muscle Artifacts from the EEG: A Review and Recommendations,” IEEE Sensors Journal, vol. 19, no. 14, pp. 5353-5368, July 2019.

Copyright $(\odot$ by the authors. Licensee TAETI, Taiwan. This article is an open access article distributed under the terms and conditions of the Creative Commons Attribution (CC BY-NC) license (https://creativecommons.org/licenses/by-nc/4.0/). 